\title{
Cesarean Scar Pregnancy: A Report of 11 Cases and Review of the Literature Regarding Subsequent Pregnancy
}

\author{
Chiaki Heshiki', Keiko Mekaru', Maho Miyagi', Sugiko Oishi' ${ }^{1}$, Kozue Akamine', \\ Hitoshi Sugiyama', Tadatsugu Kinjo', Hitoshi Masamoto', Yoichi Aoki1 \\ ${ }^{1}$ Department of Obstetrics and Gynecology, Graduate School of Medical Science, University of the Ryukyus, \\ Nishihara, Okinawa, Japan \\ ${ }^{2}$ Science and Technology Group, Okinawa Institute of Science and Technology Graduate University, Onna, \\ Okinawa, Japan \\ Email: h052400@med.u-ryukyu.ac.jp
}

Received 4 December 2015; accepted 8 January 2016; published 11 January 2016

Copyright (C) 2016 by authors and Scientific Research Publishing Inc.

This work is licensed under the Creative Commons Attribution-NonCommercial International License (CC BY-NC).

http://creativecommons.org/licenses/by-nc/4.0/

(c) () (9) Open Access

\section{Abstract}

Background: There is no consensus regarding the optimal treatment for cesarean scar pregnancy (CSP) because treatment efficacy, safety, and the influence on subsequent pregnancy must be taken into consideration. Here we report our experience with 11 cases of CSP and review the literature regarding subsequent pregnancy. Methods: Records of 11 CSP cases that were treated at our hospital were retrospectively reviewed. CSP was treated by local methotrexate (MTX) injection or laparotomic or laparoscopic removal of the gestational mass and myometrial repair. Outcome of subsequent pregnancy after treatment was followed-up until delivery. Results: Local MTX injection was performed for six cases, laparotomic removal of the gestational mass and myometrial repair was performed for two, and laparoscopic removal of the gestational mass and myometrial repair was performed for three. The uterus was preserved in all cases. After CSP treatment, eight pregnancies occurred in five cases, resulting in six live births and two miscarriages. Conclusion: Advantages and disadvantages of various treatment methods for CSP continue to be elucidated. Serum hCG level, location of the gestational mass, thickness of the lower uterine segment at the time of diagnosis, and whether the patient wishes for fertility preservation should be considered when choosing a treatment plan.

\section{Keywords}

Cesarean Scar Pregnancy, Methotrexate, Fertility Preservation 


\section{Introduction}

Cesarean scar pregnancy (CSP) is a rare form of ectopic pregnancy associated with a poor prognosis and a greater risk for uterine rupture and massive genital bleeding with an incidence of one case per $1800-2216$ pregnancies. However, the incidence has tended to increase with the continued escalation in the cesarean section rate [1]. It is important to begin treatment as soon as possible since CSP can be a life-threatening condition even at early gestational ages, although there is currently no consensus on an appropriate treatment modality even though some studies have reported successful results with methotrexate (MTX) therapy, surgical removal of the ectopic pregnancy, or uterine artery embolization [2] [3]. Frequent use of MTX therapy or surgical interventions and their good prognosis are often reported, whereas D\&C alone is considered as contraindication because of its risk of hemorrhage and uterine rupture. We typically choose local MTX injection because it is the least invasive option. Over our 14 years of experience, we have encountered two cases that required additional treatment because of unfavorable progress after local MTX injection and to preserve fertility for subsequent pregnancies. This experience has led us to reconsider our treatment method. Here we report our experience with 11 cases of CSP and review the literature regarding subsequent pregnancy.

\section{Methods}

The medical records of 11 cases of CSP that were treated at the Department of Obstetrics and Gynecology, University of the Ryukyus (Nishihara, Japan) from January 2000 to April 2015 were retrospectively reviewed. All diagnoses were made by confirmation of serum human chorionic gonadotropin (hCG) levels and ultrasonographic landmarks, including 1) the presence of a gestational sac in the anterior part of the uterus isthmus, 2) an empty uterine cavity without contact with the sac, and 3) a clearly visible empty cervical canal without contact with the sac. Until 2012, local MTX injection was the standard treatment at our facility with laparotomic or laparoscopic removal and repair of the uterine scar in cases with extremely high serum hCG levels $(>50,000$ $\mathrm{mIU} / \mathrm{mL}$ ), unstable vital signs, or massive genital/abdominal bleeding. Pregnancy outcome after treatment was followed-up until delivery in all cases.

\subsection{Local MTX Injection}

Before local MTX injection, possible complications of bleeding, uterine rupture, and future hysterectomy risk were explained to each patient. After receiving informed consent, transvaginal sonographically guided aspiration of the gestational sac and injection of MTX (50 mg/body) under intravenous or local anesthesia were performed using a $18 \mathrm{G}$ needle that was originally developed for oocyte retrieval (Univer, Tokyo, Japan).

\subsection{Laparoscopic Removal of the Gestational Mass and Myometrial Repair}

Before performing laparoscopic surgery, possible complications were explained to each patient. Each procedure was performed under general and epidural anesthesia in the Trendelenburg position. A 12-mm operative trocar was inserted into the abdominal cavity for the laparoscope and three 5-mm trocars were inserted into the pelvic cavity at the levels of the bilateral anterior superior iliac spine and suprapubically at the midline. Intra-abdominal pressure was maintained by carbon dioxide insufflation at $8 \mathrm{mmHg}$. The serosa in the vesico-uterine pouch was incised and then the bladder was exfoliated to detect the cesarean scar. Dilute vasopressin $(1 \mathrm{U} / \mathrm{mL})$ was injected into the myometrium at the site of CSP to maintain hemostasis. The CSP mass was incised, and the gestational sac was removed to an Endobag ${ }^{\mathrm{TM}}$ specimen container. The thin wall of the myometrium was incised and trimmed to expose fresh myometrium and then the incision was closed using a double layer of continuous absorbable sutures.

\subsection{Follow-Up}

Symptoms and serum hCG levels were carefully observed after MTX treatment for ectopic pregnancy (most received a single-dose regimen) to assess the necessity of additional treatment. Serum hCG levels were evaluated on days 4 and 7, and thereafter when necessary, as described elsewhere [4] [5]. Additional treatment was indicated if the serum hCG level did not decrease by at least $15 \%$ from day 4 to day 7 or did not continue to decrease after day 7. Follow-up was continued until the serum hCG level decreased. Subsequent pregnancy was permissible for those that completed follow-up. 


\section{Results}

The clinical characteristics of the 11 cases of CSP that were treated at our hospital from January 2000 to April 2015 are shown in Table 1. Local MTX injection was performed until 2012 basically because it is the least invasive of current options. Local MTX injection was administered for six cases, laparotomic removal of the gestational mass and myometrial repair was performed for two, and laparoscopic removal of the gestational mass and myometrial repair was performed for three. The postoperative course was good in five cases that were surgically managed, where as two (33\%) of six cases that received local MTX injection as a first-line treatment had very high serum hCG levels (28,892 and 34,165 mIU/mL, respectively) and required additional treatment, with one requiring emergency open surgery due to severe bleeding. The follow-up period until the serum hCG level decreased ranged from 36 to 55 days. The uterus was preserved in all cases.

After CSP treatment, eight pregnancies occurred in five cases, which included six live births through elective cesarean section at 36 weeks of gestation or later and two miscarriages (Table 2). To determine whether the thickness of the lower uterine segment in subsequent pregnancy was influenced by the location of the gestational sac at the time of diagnosis of CSP, the cases were divided into two groups according to whether the gestational sac was located between the cesarean scar and bladder (bladder side) or between the cesarean scar and endometrium (endometrium side) (Figure 1). Three cases belong to the bladder side (case 1, 2, and 4) and two belong to the endometrium side (case 3 and 5). In case 4 (bladder side), the gestational mass was bulging on the bladder side. She received local MTX injection and required no further treatment, but the lower uterine segment was thin during the subsequent pregnancy in contrast to cases 1 and 2, which underwent surgery as a first-line or additional treatment, even though the gestational masses were located on the bladder side, as with case 4 . In cases 3 and 5 (endometrium side), the gestational masses were located near the endometrium; however, the distance between the bladder and gestational mass was not considered to be short. Thinning of the lower uterine segment during the subsequent pregnancy was not confirmed in case 3 . The lower uterine segment during the subsequent

Table 1. Clinical characteristics of the 11 cases of CSP.

\begin{tabular}{cccccccc}
\hline Case & Age & Parity & GA & Serum hCG $(\mathrm{mIU} / \mathrm{mL})$ & Primary treatment & Additional treatment & Follow-up period (days) \\
\hline 1 & 29 & $1 \mathrm{G} 1 \mathrm{P}$ & 7 & 93,892 & Laparoscopy & None & 51 \\
2 & 31 & GG1P & 6 & 34,165 & MTX & Laparoscopy & 42 \\
3 & 29 & $1 \mathrm{G} 1 \mathrm{P}$ & 6 & 28,892 & MTX & Laparotomy & 55 \\
4 & 28 & $4 \mathrm{G} 3 \mathrm{P}$ & 7 & 28,300 & MTX & None & 42 \\
5 & 26 & 2G2P & 8 & 12,770 & MTX & None & 47 \\
6 & 32 & 2G2P & 6 & 4970 & MTX & None & 41 \\
7 & 37 & $5 \mathrm{G} 2 \mathrm{P}$ & 4 & 6488 & MTX & None & 48 \\
8 & 35 & $3 G 3 P$ & 7 & 18,527 & Laparotomy & None & 36 \\
9 & 36 & 1G1P & 10 & $>100,000$ & Laparotomy & None & 45 \\
10 & 29 & $6 G 3 P$ & 6 & 42,403 & Laparoscopy & None & 40 \\
11 & 35 & 2G2P & 7 & 33,592 & Laparoscopy & None & 45
\end{tabular}

Table 2. Clinical characteristics of the 5 cases of subsequent pregnancy.

\begin{tabular}{|c|c|c|c|c|c|c|}
\hline Case & $\begin{array}{c}\text { Location of } \\
\text { GS }\end{array}$ & $\begin{array}{l}\text { Primary } \\
\text { treatment }\end{array}$ & $\begin{array}{l}\text { Additional } \\
\text { treatment }\end{array}$ & $\begin{array}{l}\text { Period from treatment } \\
\text { until subsequent pregnancy } \\
\text { (months) }\end{array}$ & $\begin{array}{l}\text { Outcome of } \\
\text { subsequent } \\
\text { pregnancy }\end{array}$ & $\begin{array}{c}\text { Lower uterine segment } \\
\text { during subsequent } \\
\text { pregnancy }\end{array}$ \\
\hline 1 & Bladder s1ide & Laparoscopy & None & $\begin{array}{l}12 \\
39\end{array}$ & $\begin{array}{l}\text { GA36w C/S } \\
\text { GA38w C/S }\end{array}$ & $\begin{array}{l}\text { Thinning (-) } \\
\text { Thinning }(-)\end{array}$ \\
\hline 2 & Bladder side & MTX & Laparoscopy & $\begin{array}{c}7 \\
14\end{array}$ & $\begin{array}{l}\text { Miscarriage } \\
\text { GA38w C/S }\end{array}$ & $\begin{array}{c}\text { NA } \\
\text { Thinning (-) }\end{array}$ \\
\hline 3 & Endometrial side & MTX & Laparotomy & $\begin{array}{l}12 \\
25\end{array}$ & $\begin{array}{l}\text { GA37w C/S } \\
\text { GA38w C/S }\end{array}$ & $\begin{array}{l}\text { Thinning }(-) \\
\text { Thinning }(-)\end{array}$ \\
\hline 4 & Bladder side & MTX & None & 8 & GA38w C/S & Thinning (+) \\
\hline 5 & Endometrial side & MTX & None & 10 & Miscarriage & NA \\
\hline
\end{tabular}




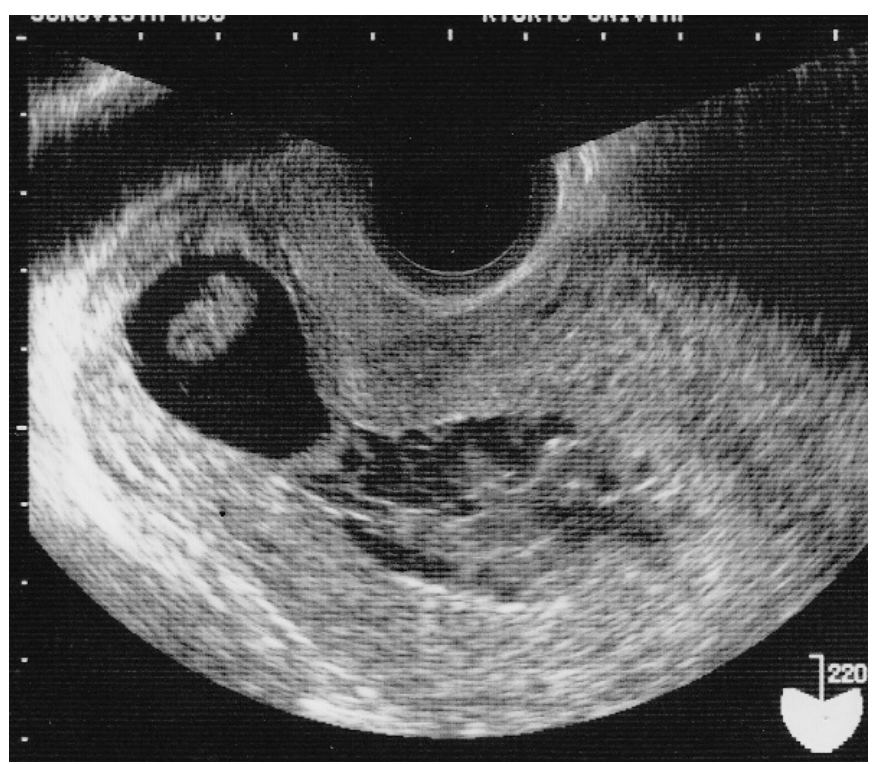

Bladder side (Case No. 4)

The gestational mass was bulging on the bladder side.

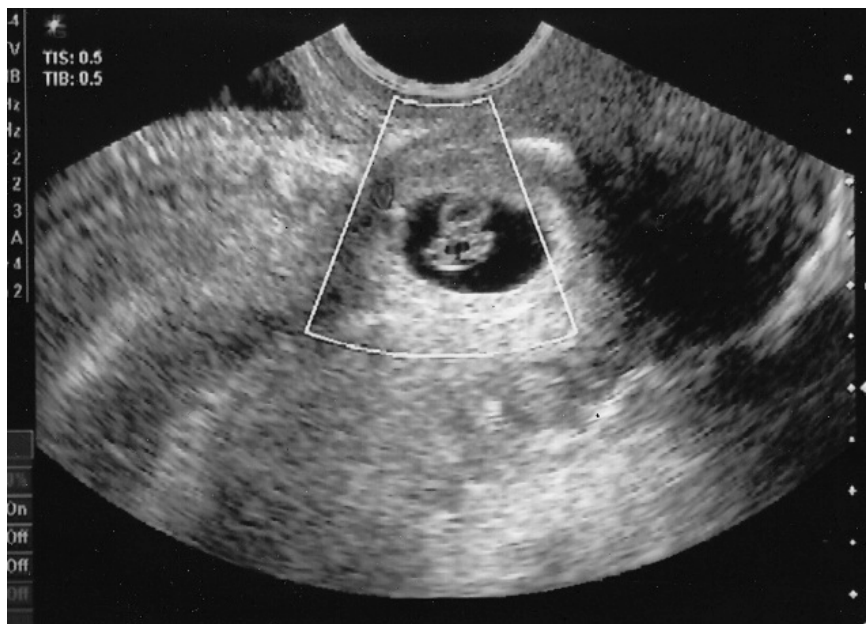

Endometrium side (Case No. 5)

The gestational mass was located near the endometrium. The distance between the bladder and gestational mass was not considered as short.

Figure 1. Location of the gestational mass at diagnosis.

pregnancy was not assessed in case 5 because the pregnancy ended in miscarriage. There were no obstetrical complications in any of the live births.

Local MTX injection was chosen as a first-line treatment because it is the least invasive of current options. However, our experience with this management scheme has raised some issues, such as 1) a longer duration to achieve a decrease in serum hCG, particularly among cases with exceptionally high serum hCG levels, which may result in prolonged hospitalization and follow-up and 2) an increased risk of obstetrical complications associated with a thin lower uterine segment in subsequent pregnancy. Therefore, we presumed that laparoscopic removal of the gestational mass and myometrial repair for such cases (i.e., remarkably high serum hCG level and thin lower uterine segments) may be associated with a decreased risk for additional treatment and uterine rupture in subsequent pregnancy.

In accordance with this point of view, laparoscopic removal of the gestational mass and myometrial repair was performed for case 10, which had a serum hCG level of $42,403 \mathrm{mIU} / \mathrm{mL}$ at the time of diagnosis and a gestational sac with a fetal heartbeat in the cesarean scar and an empty uterine cavity, as confirmed by ultrasono- 
graphy. The gestational sac projected to the bladder side so that the myometrium of the lower uterine segment became remarkably thin (Figure 2). Laparoscopic surgery was selected for this case because of the extremely high serum hCG level and thin myometrium of the lower uterine segment. A 12-mm trocar was inserted at the umbilicus into the abdominal cavity for the laparoscope. Then, three 5-mm trocars were inserted at the levels of the bilateral anterior superior iliac spine and suprapubic midline. There was no intra-abdominal bleeding, although part of the vesico-uterine pouch had a bulge due to CSP. Although the urinary bladder rigidly adhered to the lower uterine corpus and cervix, adhesiolysis was possible under a high-power field of vision. The cesarean scar was identified as thinning myometrium after the serosa of vesico-uterine pouch was incised, which was so thin that the gestational sac was observable through it (Figure 3). The urinary bladder was sufficiently pushed down to maintain an adequate margin to place sutures during the myometrial repair. After local injection of diluted vasopressin into the myometrium, CSP was incised using a unipolar knife and the gestational sac was removed with forceps. The thinning myometrium was trimmed as necessary to expose fresh myometrium and then a double layer of continuous sutures was placed in the uterine wall using 2 - 0 polyglactin. The gestational sac was removed using a specimen retrieval bag. The surgical duration was $4 \mathrm{~h}$ and $14 \mathrm{~min}$, and the total blood loss was $100 \mathrm{~g}$. The patient was discharged on postoperative day 4. On postoperative day 43, the serum hCG level decreased and an adequate thickness of the myometrium was confirmed by transvaginal ultrasonography.

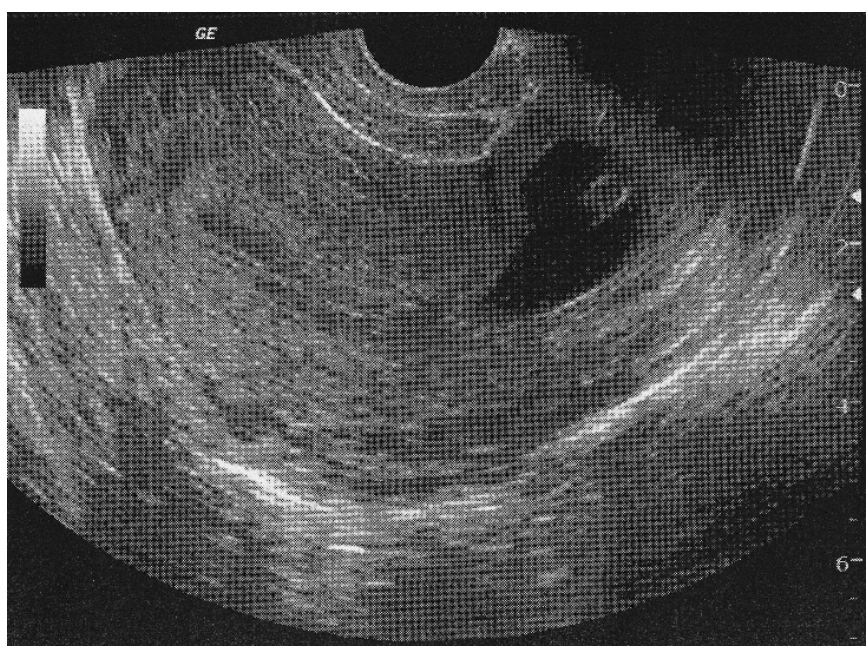

Figure 2. Ultrasonography finding at the diagnosis (case 10). The uterine cavity was empty, and gestational mass was projecting to bladder side so that the myometrium of the lower segment of the uterus became remarkably thin.

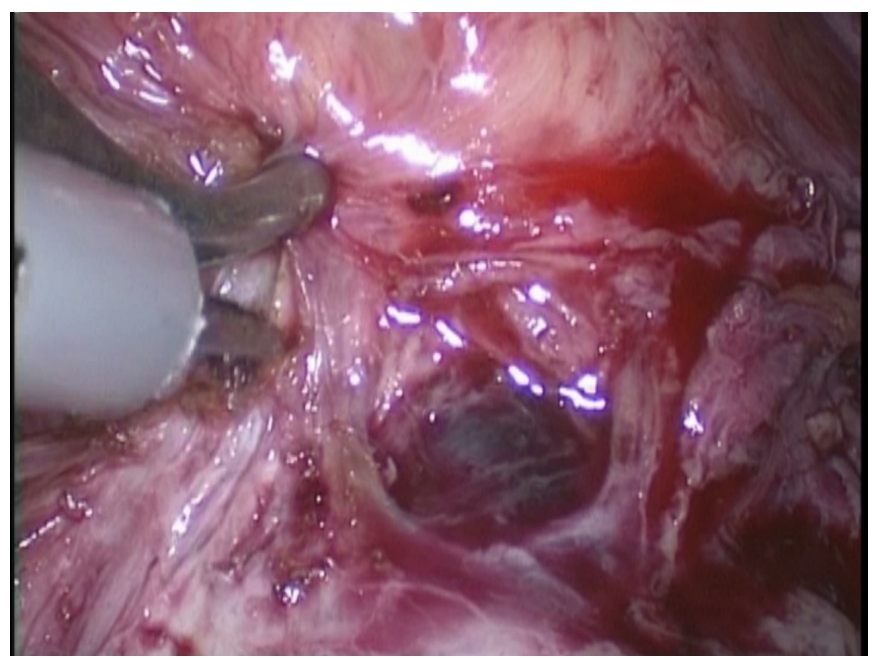

Figure 3. Cesarean scar after incision of the serosa of the vesico-uterine pouch. The cesarean scar was identified as a thin myometrium after incision of the serosa of the vesico-uterine pouch that allowed visualization of gestational sac. 


\section{Discussion}

The incidence of CSP is estimated at one case per 1800 - 2216 pregnancies and approximately $6.1 \%$ of patients have a history of cesarean section [3]. There is no consensus on an optimal treatment for CSP because of its rarity; therefore, various treatment modalities have been employed, including local MTX injection, surgical removal of the gestational mass, or uterine artery embolization [2] [3]. Local MTX injection is the least invasive of these options and can be performed easily and immediately. However, serum hCG levels following local MTX injection decrease slower than those following surgical treatment. Therefore, there may be risks of prolonged bleeding, hospitalization, and follow-up in addition to the necessity of additional treatment if the decrease in serum hCG is unsatisfactory. Furthermore, if the myometrium at the lower uterine segment after treatment of CSP remains thin during the subsequent pregnancy, then the increased risk of uterine rupture or recurrence of CSP remains a significant concern [6] [7]. Surgical removal of the gestational mass and myometrial repair is more invasive than medical treatment with MTX, but a laparoscopic approach can minimize invasiveness. Laparoscopic surgery is associated with a decreased need for additional treatment, a shorter follow-up period can be expected, and thinning of the myometrium at the lower uterine segment during subsequent pregnancy may be avoided following myometrial repair [8] [9].

In a review by Rotas et al., 15 cases were managed by local MTX injection, with eight receiving only a single injection, although several months were required to achieve a curative effect, while the remaining seven cases required additional treatment. In 12 cases, the gestational mass was removed via laparotomy or laparoscopy. Of these cases, 11 required no additional treatment, and the follow-up period was shorter than that with medical treatment [2]. Until 2012, local MTX injection was the preferred treatment in our hospital for cases in stable condition and serum hCG levels of $<50,000 \mathrm{mIU} / \mathrm{mL}$, as described in a previous report [10]. Even though we carefully screened patients, two cases had markedly elevated serum hCG levels and required additional treatment. In addition, hemorrhaging occurred during treatment in one case. Thinning of the myometrium at the lower uterine segment during subsequent pregnancy was confirmed in one case following local MTX injection, and consequently, cautious follow-up and observation were required until delivery. After reconsidering our treatment policy from these experiences, we decided to perform laparoscopic removal of the gestational mass and repair the thin myometrium to reduce the risk of additional treatment and shorten the follow-up period for cases with high serum hCG levels.

Recent reports regarding subsequent pregnancy after treatment of CSP are listed in Table 3. Wang et al. reported an advantage of surgical removal of the gestational mass and myometrial repair because thin myometrium at the level of the cesarean scar can increase the risk of CSP recurrence or uterine rupture during subsequent pregnancy [8]. However, Nagi et al. reported fertility preservation in 37 cases, which included 9 that received local MTX injection and 28 treated by dilatation and curettage (D\&C). Of 24 cases that wished for fertility preservation after CSP, 21 (87.5\%) conceived naturally. Recurrence of CSP developed in only one case, while none developed other obstetrical complications, such as uterine rupture, placenta previa, or placenta accreta. Therefore, they concluded that myometrial repair is not always necessary [11]. Although, the location of the gestational sac and thickness of the lower uterine segment at the time of diagnosis of CSP were not discussed in this study, one case of recurrent CSP with a large uterine defect that extended into the lower part of the uterine corpus was reported. Seow et al. reported two cases of placenta accrete among six live births and one case of uterine rupture that was treated with D\&C, which resulted in maternal death [12]. It is conceivable that the unrecovered thin myometrium after CSP treatment may have caused uterine rupture in this case. Currently, it is thought that D\&C alone is contraindicated for CSP because it is obviously associated with severe maternal complications. However, Zhuang and Huang reported a favorable outcome for D\&C preceded by uterine artery embolization [13]. Maymon et al. reported six cases of subsequent pregnancies following CSP (seven deliveries in total), including two cases of recurrent CSP in two live births delivered by emergency cesarean section because of placenta abruption at gestational week 34 and acute fetal distress at gestational week 41 [14]. Apart from these cases, Vial et al. reported one live birth after CSP treated by laparotomy [15] and Korkontzelos et al. reported one case of subsequent pregnancy after CSP treated with hysteroscopic MTX injection, although this report included no information regarding the location of the gestational sac or thinning of the lower segment during subsequent pregnancy [16]. In our study, most subsequent pregnancies, with the exception of two miscarriages, resulted in live births by elective cesarean section after gestational week 36 without uterine rupture or placental abnormalities. Nevertheless, there is currently no consensus on an optimal management regimen because there is no agreement among the opinions of various researchers. 
Table 3. Recent reports regarding subsequent pregnancy after treatment of CSP.

\begin{tabular}{|c|c|c|c|c|}
\hline Report & $\begin{array}{l}\text { No. of } \\
\text { cases }\end{array}$ & $\begin{array}{c}\text { Method of } \\
\text { conservative treatment }\end{array}$ & Outcome of CSP treatment & Subsequent pregnancy \\
\hline $\begin{array}{l}\text { Nagi } \\
(2007)\end{array}$ & 40 & $\begin{array}{l}\text { 28: D\&C } \\
\text { 9: MTX } \\
\text { 3: Expectant }\end{array}$ & $\begin{array}{l}\text { D\&C: Fertility preserved for all cases } \\
\text { MTX: 3/9 required additional treatment, } \\
\text { fertility preserved for all cases } \\
\text { Expectant: } 2 / 3 \text { required hysterectomy, } \\
\text { fertility preserved for } 1 \text { case }\end{array}$ & $\begin{array}{l}21 \text { cases of clinical pregnancies } \\
\text { 9: Live birth } \\
\text { 7: Miscarriage } \\
\text { 2: Ongoing pregnancy } \\
\text { 1: Recurrence of CSP } \\
\text { 2: Unknown }\end{array}$ \\
\hline $\begin{array}{l}\text { Seow } \\
(2004)\end{array}$ & 15 & $\begin{array}{l}\text { D\&C } \\
\text { Laparotomy } \\
\text { MTX }\end{array}$ & Fertility preserved for 14 cases & $\begin{array}{l}7 \text { cases of clinical pregnancy } \\
\text { 6: Live birth ( } 2 \text { cases of placenta } \\
\text { accreta) } \\
\text { 1: Death due to uterine rupture at } 38 \\
\text { weeks (treated by D\&C) }\end{array}$ \\
\hline $\begin{array}{l}\text { Maymon } \\
\text { (2011) }\end{array}$ & 18 & $\begin{array}{l}\text { 12: MTX } \\
\text { 2: Laparotomy } \\
\text { 2: Laparotomy after } \\
\text { MTX failed }\end{array}$ & Fertility preserved for all cases & $\begin{array}{l}8 \text { cases of clinical pregnancy } \\
\text { 2: Recurrent CSP } \\
\text { 6: Live birth ( } 6 \text { cases, } 7 \text { deliveries } \\
\text { altogether) } \\
\text { 5: Term elective C/S } \\
\text { 2: Emergency C/S ( } 41 \text { weeks, acute } \\
\text { fetal distress and } 34 \text { weeks, abruption) }\end{array}$ \\
\hline $\begin{array}{l}\text { Heshiki } \\
\text { (2015) }\end{array}$ & 11 & $\begin{array}{l}\text { 6: MTX } \\
\text { 3: Laparoscopy } \\
\text { 2: Laparotomy }\end{array}$ & Fertility preserved for all cases & $\begin{array}{l}8 \text { cases of clinical pregnancy } \\
\text { 6: Live birth } \\
\text { 2: Miscarriage }\end{array}$ \\
\hline
\end{tabular}

\section{Conclusion}

Surgical removal of the gestational mass for CSP will facilitate simultaneous repair of the myometrium and may decrease the risk of perinatal complications during a subsequent pregnancy. The advantages and disadvantages of various treatment methods should be carefully reviewed to choose an optimal treatment plan by taking into account the serum hCG level, location of the gestational mass, thickness of the lower uterine segment at the time of diagnosis, and whether the patient wishes to preserve fertility.

\section{References}

[1] Jurkovic, D., Hillaby, K., Lawrence, A., Salim, R. and Elson, C.J. (2003) First-Trimester Diagnosis and Management of Pregnancies Implanted into the Lower Segment Cesarean Section Scar. Ultrasound in Obstetrics \& Gynecology, 21, 220-227. http://dx.doi.org/10.1002/uog.56

[2] Rotas, M.A., Haberman, S. and Levgur, M. (2006) Cesarean Scar Ectopic Pregnancies. Obstetrics \& Gynecology, 107, 1373-1381. http://dx.doi.org/10.1097/01.AOG.0000218690.24494.ce

[3] Ash, A., Smith, A. and Maxwell, D. (2007) Caesarean Scar Pregnancy. BJOG, 114, 253-263. http://dx.doi.org/10.1111/j.1471-0528.2006.01237.x

[4] Speroff, L. and Frits, M.A. (2005) Clinical Gynecologic Endocrinology and Infertility. Lippincott Williams and Wilkins, Philadelphia, 1285-1302.

[5] American College of Obstetricians and Gynecologists (2008) ACOG Practice Bulletin No. 94: Medical Management of Ectopic Pregnancy. Obstetrics \& Gynecology, 111, 1479-1485. http://dx.doi.org/10.1097/AOG.0b013e31817d201e

[6] Halperin, R., Schneider, D., Mendlovic, S., Pansky, M., Herman, A. and Maymon, R. (2009) Uterine-Preserving Emergency Surgery for Cesarean Scar Pregnancies: Another Medical Solution to an Iatrogenic Problem. Fertility and Sterility, 91, 2623-2627. http://dx.doi.org/10.1016/j.fertnstert.2008.03.021

[7] Seow, K.M., Wang, P.H., Huang, L.W. and Hwang, J.L. (2013) Transvaginal Sono-Guided Aspiration of Gestational Sac Concurrent with a Local Methotrexate Injection for the Treatment of Unruptured Cesarean Scar Pregnancy. Archives of Gynecology and Obstetrics, 288, 361-366. http://dx.doi.org/10.1007/s00404-013-2765-4

[8] Wang, Y.L., Su, T.H. and Chen, H.S. (2006) Operative Laparoscopy for Unruptured Ectopic Pregnancy in a Cesarean Scar. BJOG, 113, 1035-1038. http://dx.doi.org/10.1111/j.1471-0528.2006.01031.x

[9] Fylstra, D.L. (2002) Ectopic Pregnancy within a Cesarean Scar: A Review. Obstetrical \& Gynecological Survey, 57, 537-543. http://dx.doi.org/10.1097/00006254-200208000-00024 
[10] Yang, X.Y., Yu, H., Li, K.M., Chu, Y.X. and Zheng, A. (2010) Uterine Artery Embolization Combined with Local Methotrexate for Treatment of Cesarean Scar Pregnancy. BJOG, 117, 990-996. http://dx.doi.org/10.1111/j.1471-0528.2010.02578.x

[11] Nagi, J.B., Helmy, S., Yebovi, D.O., Yazbek, J., Sawyer, E. and Jurkovic, D. (2007) Reproductive Outcomes of Women with a Previous History of Cesarean Scar Ectopic Pregnancies. Human Reproduction, 22, 2012-2015. http://dx.doi.org/10.1093/humrep/dem078

[12] Seow, K.M., Hwang, J.L., Tsai, Y.L., Huang, L.W., Lin, Y.H. and Hsieh, B.C. (2004) Subsequent Pregnancy Outcome after Conservative Treatment of a Previous Cesarean Scar Pregnancy. Acta Obstetricia et Gynecologica Scandinavica, 83, 1167-1172. http://dx.doi.org/10.1111/j.0001-6349.2004.00445.x

[13] Zhuang, Y. and Huang, L. (2009) Uterine Artery Embolization Compared with Methotrexate for the Management of Pregnancy Implanted within a Cesarean Scar. American Journal of Obstetrics and Gynecology, 201, 152e1-152e3.

[14] Maymon, R., Svirsky, R., Smorgick, N., Mendlovic, S., Halperin, R., Gilad, K., et al. (2011) Fertility Performance and Obstetric Outcome among Women with Previous Cesarean Scar Pregnancy. Journal of Ultrasound in Medicine, $\mathbf{3 0 ,}$ 1179-1184.

[15] Vial, Y., Patignat, P. and Hohfeld, P. (2000) Pregnancy in a Cesarean Scar. Ultrasound in Obstetrics and Gynecology, 16, 592-593. http://dx.doi.org/10.1046/j.1469-0705.2000.00300-2.x

[16] Korkontzelos, I., Panagiotis, T., Nikolaos, A., Souliotis, D. and Kosmas, I. (2008) Successful Term Pregnancy after Treatment of a Cesarean Scar Ectopic Gestation by Endoscopic Technique and Conservative Therapy. Fertility and Sterility, 90, 2010e13-15. http://dx.doi.org/10.1016/j.fertnstert.2008.04.027 